Journal of Advanced Research in Fluid Mechanics and Thermal Sciences

\title{
Numerical Solution for Unsteady Acceleration MHD Third-Grade Fluid Flow in a Rotating Frame Through Porous Medium Over Semi-Infinite Boundary Condition with a Presence of Heat Transfer
}

\author{
Shafaruniza Mahadi ${ }^{1,}{ }^{*}$, Yeak Su Hoe ${ }^{2}$, Norazam Arbin ${ }^{1}$, Faisal Salah ${ }^{3,4}$ \\ Faculty of Computer and Mathematical Sciences, Universiti Teknologi MARA, Cawangan Johor, Kampus Segamat, Johor, Malaysia \\ Department of Mathematical Sciences, Faculty of Science, Universiti Teknologi Malaysia, 81318 Johor Bahru, Johor, Malaysia \\ Department of Mathematics, College of Science and Arts, King Abdul-Aziz University, Rabigh, Saudi Arabia \\ Department of Mathematics, Faculty of Science, University of Kordofan, Elobiad, Sudan
}

\section{Article history:}

Received 8 April 2021

Received in revised form 1 August 2021

Accepted 2 August 2021

Available online 20 September 2021

\section{Keywords:}

Hybrid method; rotating frame; porous medium; magnetohydrodynamic; heat transfer; asymptotic interpolation

\begin{abstract}
The aim of this work is to present a suitable numerical solution for unsteady nonNewtonian third-grade fluid which rotates at $z$-axis and pass through a porous medium. The fluid flows in magnetic field with constant acceleration and the semiinfinite boundary condition are highlighted. The fluid problem is also deal with heat transfer. The nonlinear partial differential equation is discretised using the finite difference method (FDM). The linear system obtained for three different domains (lengths). Consequently, the asymptotic interpolation method is merged to solve problems of large sizes. This hybrid method yielded results that satisfied the boundary condition that reaches zero as length grows to infinite length. For velocity profile and temperature distribution, a comparison of FDM and hybrid method is shown. It is discovered that the hybrid method produces better results than FDM for this infinitely large problem. Several analyses have been carried out to investigate the effect of various fluid parameter values. The findings reveal that as the porosity parameter increases, the velocity decreases. The Grashof and Prandtl numbers demonstrate the relationship to the temperature distributions. The effects of the magnetic field and the non-Newtonian parameters were also illustrated, as these parameters influence the velocity distribution of the fluid flow.
\end{abstract}

\section{Introduction}

Recently, application of non-Newtonian fluid problem in a various of disciplines, covering meteorology, geophysics, engineering, and mathematics have been notified [1-3]. The nonNewtonian equations offer difficulties for researchers because they are more complex than those of Newtonian fluid.

The finite difference method (FDM) is the most well numerical method that most researchers use to find the numerical solution especially in fluid flow problems. The FDM substitutes difference

\footnotetext{
* Corresponding author.

E-mail address: shafa669@uitm.edu.my
}

https://doi.org/10.37934/arfmts.87.2.90105 
quotients for derivatives in the governing field equations, which include values of the solution at discrete mesh points in the domain under study where this is called discretisation. This representation is used repeatedly to create algebraic systems of equations in terms of unknown mesh point values. The main problem with this system is the inaccuracy in dealing with regions of complicated domain, even though it can be solved using coordinate transformation techniques $[4,5]$.

Tome [6] applied the FDM to address two-dimensional modified Newtonian flow with several free surfaces within an arbitrary domain. After eight years, the problem has been extended to threedimensional modified Newtonian free surface flows with additional features, and it has been resolved using the same mathematical approach [7]. The numerical method of implicit FDM is used to convert partial differential equations to a system of non-linear equations by using the damped-Newton method to solve non-Newtonian third-grade fluid which pass a porous medium [8]. The MHD rotating flow equations of fourth-grade fluid bounded between two non-conducting rigid plates in a rotating frame with uniform transverse magnetic field is modelled. The second-order Central difference formula and successive under relaxation scheme have been used to solve the equation. The finding reveals that the flow behaviour is parameter dependent [9].

Parida and Padhy [10] used the FDM in 2018 to discretise the differential equation of MHD thirdgrade in a non-Darcian porous medium. Garg [11] and Anitha [12] have explored the chemical reaction with radiation in greater details. Nayak [13] performed an investigation of heat transfer exists in fluid flow with the issue of focusing on differential form of MHD third grade with viscous dissipation. FDM is used to solve the fluid problem. According to the results, FDM is still relevant today as it can provide an approximate system for solving nonlinear differential equations of fluids.

The implicit scheme finite difference Keller-box approach was used to address the problem of MHD incompressible viscous fluid between two parallel porous plates and velocity slip boundary conditions at the permeable boundaries [14]. The Keller-box approach also been used to evaluate heat transfer and the effects of an external magnetic field on the non-Newtonian micropolar fluid flow between a porous and a non-porous disc [15].

MHD also called magnetic field fluid dynamics, is the analysis of the dynamics of electrically conducting fluids, like salt water and electrolytes [16]. Interactions exist between the motion in fluid flow where electric current is generated when fluid flows across magnetic lines and the transverse magnetic lines of forces which lead to other factors on fluid. This present work also focuses on other two parameters which are rotating frame and a porous medium. A rotating frame is one that rotates with respect to an inertial reference frame. Fluid flow in a porous media has recently emerged as a research subject of interest. Porous medium is a parameter in the momentum equation that applies to porous materials such as rocks or soil. Several works have been published, including the development of new exact solutions for MHD transient Rotating flow of a second-grade fluid in a porous medium [2,17], third-grade fluid in a porous medium and a rotating frame [18], MHD rotating flow of a fourth-grade fluid [8] and MHD flow of a third-order fluid in a porous medium [19].

Heat transfer in non-Newtonian fluids can be seen in many industrial processes, such as the plastic manufacturing process. Aiyesami [20] investigated the fluid motion with temperature for the MHD third-grade fluid using an semi-analytical solution called HAM. El-dabe [21] used homotopy perturbation to solve an MHD boundary-layer flow of an incompressible fourth-grade nanofluid through a porous medium down a vertical cylinder with heat transfer.

The aim of this paper is to solve a third-grade non-Newtonian fluid flows with constant acceleration in a rotating frame across a magnetic field and porous medium with thermal conductivity. The nonlinear differential equation is discretised using FDM. Since the fluid problem has a semi-infinite boundary domain which present an infinite length, a hybrid method of finite difference - asymptotic interpolation method is designed and applied to the problem system. 
Asymptotic interpolation method is implemented to boost the result where this method has been applied in mechanical and chemical hydrodynamics [22,23] and investigating fluctuating fluid membranes to give accurate predictions at infinity [24]. The hybrid method has previously been used to solve MHD third grade fluid in a rotating frame $[25,26]$. The discussions are centred on the comparison between classical FDM and hybrid method, and the effect of velocity profile and temperature distribution with varying dimensionless parameter values.

\section{Mathematical Formulation}

Define the velocity field, $V=(u(z, t), v(z, t), w(z, t))$ that exists in fluid where $u, v$ and $w$ are the velocity elements that correspond to the $x$-axis, $y$-axis and $z$-axis. The equation of continuity is given by

$\nabla \cdot V=0$.

The following equations govern the MHD fluid flow in a porous medium and a rotating frame

$$
\left(\frac{\partial V}{\partial t}+(V \cdot \nabla) V+2 \Omega \times V+\Omega \times(\Omega \times r)\right)=-\nabla p+\operatorname{div} T-\sigma \beta_{0}{ }^{2} V+R
$$

where $\rho$ is the density, $p$ is the pressure, $r$ is the radial coordinate and $\Omega$ is the rotating parameter which rotates about $z$-axis with $V=(u(z, t), v(z, t), 0)$. Lorentz force per unit volume is given by $J \times B=-\sigma \beta_{0}{ }^{2} V$ where $J$ is current density and $B$ is total magnetic field. According to Abelman [3], the flow resistance for third grade fluid is given by

$R=-\frac{\varphi}{k}\left[\mu+\alpha_{1} \frac{\partial}{\partial t}+2 \beta_{3}\left(\left(\frac{\partial u}{\partial z}\right)^{2}+\left(\frac{\partial v}{\partial z}\right)^{2}\right)\right] V$

$T=-p I+\sum_{j=1}^{n} S_{j}$ is the stress tensor, where $p$ is force or pressure and $I$ is the identity tensor [13]. The first three tensors are given by $S_{1}=\mu A_{1}, S_{2}=\alpha_{1} A_{2}+\alpha_{2} A_{1}^{2}$ and $S_{3}=\beta_{1} A_{3}+$ $\beta_{2}\left(A_{2} A_{1}+A_{1} A_{2}\right)+\beta_{3}\left(\operatorname{tr} A_{1}^{2}\right) A_{1}$ where $\mu$ is the shear viscosity coefficient. The material constants are $\alpha_{i}(i=1,2), \beta_{j}(1,2,3), \gamma_{k}(k=1,2, \ldots, 8)$ while $A_{n}$ is the Rivlin-Ericson tensors $A_{n}=\frac{d}{d t}\left(A_{n-1}\right)+$ $A_{n-1}(\operatorname{grad} V)+(\operatorname{grad} V)^{T} A_{n-1}, n>1 \quad$ where $A_{1}=(\operatorname{grad} V)+(\operatorname{grad} V)^{T} \quad$ and $\quad A_{2}=\frac{d A_{1}}{d t}+$ $A_{1}(\operatorname{grad} V)+(\operatorname{gradV})^{T} A_{1}$. The constitutive equation for third grade fluid $(n=3)$, is $T=-p I+$ $\mu A_{1}+\alpha_{1} A_{2}+\alpha_{2} A_{1}^{2}+\beta_{1} A_{3}+\beta_{2}\left(A_{2} A_{1}+A_{1} A_{2}\right)+\beta_{3}\left(\operatorname{tr} A_{1}^{2}\right) A_{1}$ which satisfies $\mu \geq 0, \alpha_{1} \geq 0, \mid \alpha_{1}+$ $\alpha_{2} \mid \leq \sqrt{24 \mu \beta_{3}}, \beta_{1}=\beta_{2}=0, \beta_{3} \geq 0$ and $\gamma_{k}=0[3,18-20,27,28]$.

\section{Governing Equation}

The momentum equation of third grade fluid flow with heat transfer is derived as follows.

$$
\begin{aligned}
& \rho\left(\frac{\partial u}{\partial t}-2 \Omega v\right)=\mu \frac{\partial^{2} u}{\partial z^{2}}+\alpha_{1} \frac{\partial^{3} u}{\partial z^{2} \partial t}+2 \beta_{3} \frac{\partial}{\partial z}\left[\frac{\partial u}{\partial z}\left(\left(\frac{\partial u}{\partial z}\right)^{2}+\left(\frac{\partial v}{\partial z}\right)^{2}\right)\right] \\
& -\sigma \beta_{0}{ }^{2} u-\frac{\varphi}{k}\left[\mu+\alpha_{1} \frac{\partial}{\partial t}+2 \beta_{3}\left(\left(\frac{\partial u}{\partial z}\right)^{2}+\left(\frac{\partial v}{\partial z}\right)^{2}\right)\right] u+\frac{\beta g}{\rho}\left(T-T_{\infty}\right) u
\end{aligned}
$$


$\rho\left(\frac{\partial v}{\partial t}+2 \Omega u\right)=\mu \frac{\partial^{2} v}{\partial z^{2}}+\alpha_{1} \frac{\partial^{3} v}{\partial z^{2} \partial t}+2 \beta_{3} \frac{\partial}{\partial z}\left[\frac{\partial v}{\partial z}\left(\left(\frac{\partial u}{\partial z}\right)^{2}+\left(\frac{\partial v}{\partial z}\right)^{2}\right)\right]$
$-\sigma \beta_{0}{ }^{2} v-\frac{\varphi}{k}\left[\mu+\alpha_{1} \frac{\partial}{\partial t}+2 \beta_{3}\left(\left(\frac{\partial u}{\partial z}\right)^{2}+\left(\frac{\partial v}{\partial z}\right)^{2}\right)\right] v+\frac{\beta g}{\rho}\left(T-T_{\infty}\right) v$

with a simple energy equation is

$\rho C_{p} \frac{\partial T}{\partial t}=k \frac{\partial^{2} T}{\partial z^{2}}$

where $\beta$ is the coefficient of thermal expansion, $g$ is gravity, $T$ is fluid temperature, $T_{\infty}$ is ambient temperature, $C_{p}$ represents a fluid at constant pressure and $k$ is thermal conductivity. Following Nazari [29] for constant acceleration, the boundary and initial condition are

$u(0, t)=A t, v(0, t)=0, t>0, T=T_{w}$
$u(z, t) \rightarrow 0, v(z, t) \rightarrow 0$ as $z \rightarrow \infty \forall t, T \rightarrow T_{\infty}$
$u(z, 0)=0, v(z, 0)=0, z>0$.

Eq. (5) is multiplied by $i$ and added to Eq. (4). The momentum equation becomes

$\frac{\partial F}{\partial t}+2 i \Omega F$

$=v \frac{\partial^{2} F}{\partial z^{2}}+\frac{\alpha_{1}}{\rho} \frac{\partial^{3} F}{\partial z^{2} \partial t}+\frac{2 \beta_{3}}{\rho} \frac{\partial}{\partial z}\left[\left(\frac{\partial F}{\partial z}\right)^{2}\left(\frac{\partial \bar{F}}{\partial z}\right)\right]-\frac{\sigma}{\rho} \beta_{0}{ }^{2} F-\frac{\varphi}{k \rho}\left[\mu+\alpha_{1} \frac{\partial}{\partial t}+2 \beta_{3}\left(\frac{\partial F}{\partial z}\right)\left(\frac{\partial \bar{F}}{\partial z}\right)\right] F+\frac{\beta g}{\rho}(T-$

$\left.T_{\infty}\right) F$

where $F=u+i v$ and $\bar{F}=u-i v$. The boundary and initial condition now are

$$
\begin{aligned}
& F(0, t)=A t, T(z, 0)=0 \text { at } t=0 \\
& F(z, t) \rightarrow 0, T(z, t) \rightarrow T_{\infty} \text { as } z \rightarrow \infty \\
& F(z, 0)=0, T(z, 0)=0 \text { at } t=0
\end{aligned}
$$

The dimensionless parameters in this problem are $\theta^{*}=\frac{T-T_{\infty}}{T_{w}-T_{\infty}}, G r=\frac{g \beta\left(T_{w}-T_{\infty}\right) A^{3}}{v^{2}}, \operatorname{Pr}=\frac{\mu C_{P}}{k}$ where $\theta^{*}$ is the temperature of the velocity profile, $G r$ is the Grashof number and $P r$ is the Prandt number. The nonlinear equations with heat transfer are

$$
\begin{aligned}
& (1+\zeta) \frac{\partial f}{\partial \tau}+\left(2 i \Omega+M+c-G r^{*} \theta^{*}\right) f \\
& =\frac{\partial^{2} f}{\partial \eta^{2}}+a \frac{\partial^{3} f}{\partial \eta^{2} \partial \tau}+b\left[2\left(\frac{\partial^{2} f}{\partial \eta^{2}}\right)\left(\frac{\partial f}{\partial \eta}\right)\left(\frac{\partial \bar{f}}{\partial \eta}\right)+\left(\frac{\partial f}{\partial \eta}\right)^{2}\left(\frac{\partial \bar{f}}{\partial \eta}\right)\right]-2 b c f\left[\left(\frac{\partial f}{\partial \eta}\right)\left(\frac{\partial \bar{f}}{\partial \eta}\right)\right] \\
& \frac{\partial \theta}{\partial \tau}=\frac{1}{\operatorname{Pr}} \frac{\partial^{2} \theta}{\partial \eta^{2}}
\end{aligned}
$$

where $G r^{*}=\frac{G r}{\rho}\left(\frac{v^{7}}{A^{5}}\right)^{\frac{1}{3}}$. The boundary and initial conditions are 


$$
\begin{aligned}
& f(0, \tau)=\tau, \theta(0, \tau)=1 \text { for } \tau>0 \\
& f(\eta, \tau)=0, \theta(\eta, \tau)=0 \text { as } \eta \rightarrow \infty \\
& f(\eta, 0)=0, \theta(\eta, 0)=0
\end{aligned}
$$

\section{Method of Solutions}

\subsection{Finite Difference Method (FDM)}

The nonlinear equations of Eq. (10-11) are discretised using central and forward difference as follow

$$
\begin{aligned}
& \frac{(1+\zeta)}{\Delta \tau}\left(f_{i}^{j+1}-f_{i}^{j}\right)+\left(2 i \Omega+M+c-G r^{*} \theta^{*}\right) f_{i}^{j} \\
& =\frac{f_{i+1}^{j}-2 f_{i}^{j}+f_{i-1}^{j}}{\Delta \eta^{2}}+\frac{a}{\Delta \eta^{2} \Delta \tau}\left[\left(f_{i+1}^{j+1}-2 f_{i}^{j+1}+f_{i-1}^{j+1}\right)-\left(f_{i+1}^{j}-2 f_{i}^{j}+f_{i-1}^{j}\right)\right] \\
& +\frac{b}{\Delta \eta^{4}}\left(f_{i+1}^{j}-2 f_{i}^{j}+f_{i-1}^{j}\right)\left(f_{i+1}^{j}-f_{i-1}^{j}\right)\left(\bar{f}_{i+1}^{j}-\bar{f}_{i-1}^{j}\right)+\frac{b}{2 \Delta \eta^{4}}\left(f_{i+1}^{j}-f_{i-1}^{j}\right)^{2}\left(\bar{f}_{i+1}^{j}-2 \bar{f}_{i}^{j}+\bar{f}_{i-1}^{j}\right) \\
& -\frac{b c}{2 \Delta \eta} f_{i}^{j}\left(f_{i+1}^{j}-f_{i-1}^{j}\right)\left(\bar{f}_{i+1}^{j}-\bar{f}_{i-1}^{j}\right)
\end{aligned}
$$

and

$$
\frac{\theta_{i}^{j+1}-\theta_{i}^{j}}{\Delta \tau}=\frac{1}{\operatorname{Pr}}\left(\frac{\theta_{i+1}^{j}-2 \theta_{i}^{j}+\theta_{i-1}^{j}}{(\Delta \eta)^{2}}\right)
$$

where $i$ and $j$ are indices of $f\left(\eta_{i}, \tau_{j}\right)$. Bring the time-step of $j+1$ to the left side and hence the equations become

$$
\begin{aligned}
& \frac{(1+\zeta)}{\Delta \tau} f_{i}^{j+1}-\frac{a\left(f_{i+1}^{j+1}-2 f_{i}^{j+1}+f_{i-1}^{j+1}\right)}{\Delta \eta^{2} \Delta \tau} \\
& =\frac{f_{i+1}^{j}-2 f_{i}^{j}+f_{i-1}^{j}-\frac{a}{\Delta \eta^{2}}\left(f_{i+1}^{j}-2 f_{i}^{j}+f_{i-1}^{j}\right)}{\Delta \eta^{2} \Delta \tau}+\frac{b}{\Delta \eta^{4}}\left(f_{i+1}^{j}-2 f_{i}^{j}+f_{i-1}^{j}\right)\left(f_{i+1}^{j}-f_{i-1}^{j}\right)\left(\bar{f}_{i+1}^{j}-\bar{f}_{i-1}^{j}\right) \\
& +\frac{b}{2 \Delta \eta^{4}}\left(f_{i+1}^{j}-f_{i-1}^{j}\right)^{2}\left(\bar{f}_{i+1}^{j}-2 \bar{f}_{i}^{j}+\bar{f}_{i-1}^{j}\right)-\frac{b c}{2 \Delta \eta} f_{i}^{j}\left(f_{i+1}^{j}-f_{i-1}^{j}\right)\left(\bar{f}_{i+1}^{j}-\bar{f}_{i-1}^{j}\right) \\
& +\frac{(1+\zeta)}{\Delta \tau} f_{i}^{j}-\left(2 i \Omega+M+c-G r^{*} \theta^{*}\right) f_{i}^{j}
\end{aligned}
$$

and

$$
\frac{1}{\Delta \tau} \theta_{i}^{j+1}=\frac{1}{\Delta \tau} \theta_{i}^{j}+\frac{1}{\operatorname{Pr}(\Delta \eta)^{2}}\left(\theta_{i+1}^{j}-2 \theta_{i}^{j}+\theta_{i-1}^{j}\right)
$$

The initial and boundary conditions are $f_{1}^{j}=A(j-1) \Delta \tau, \theta_{1}^{n}=1$ for $j=1,2,3, \ldots, f_{L}^{n}=0, \theta_{L}^{n}=$ 0 for every $n=1,2,3, \ldots, L=L_{1}, L_{2}, L_{3}$ and $f_{i}^{1}=0$ for $i=1,2,3, \ldots, N+1$. The equation can be written in matrix form, with dimension $N-2$ 


$$
\left[A_{f}\right][f]=\left[b_{f}\right]
$$

and

$$
\left[A_{\theta}\right][\theta]=\left[b_{\theta}\right]
$$

To satisfy semi-infinite boundary condition in Eq. (12), this research investigated the fluid flow problem at three lengths which are $L=L_{1}, L_{2}, L_{3}=6,12,18$. In this paper, the step sizes are $\Delta \eta=$ $1 / 4$ and $\Delta \tau=1 / 5$ with time iteration is 6 . Since the equations involve complex functions such that in Eq. (8) and is very difficult to calculate manually, MATLAB programming is coded to find the output. Table 1 presents the velocity profile obtained from FDM with $a, b, c, \zeta, \Omega, M, G r, \operatorname{Pr}, t=1$ keep fixed. They show that the velocities start different either increase or decrease at $\eta=4$. Table 2 presents the temperature distribution where they start different at $\eta=4$. These prove that the velocity and temperature are different for different length. In order to obtain the accurate results for each node, these three lengths will be blended together in nonlinear least square in the next section.

The data from Table 1 and Table 2 are plotted to see the convergence results. Figure 1 shows the curvature of results which they are converges to the solution at each length, $L=L_{1}, L_{2}, L_{3}=$ $6,12,18$.

Table 1

The velocity profile at different lengths by FDM

\begin{tabular}{lllllll}
\hline & $\boldsymbol{u}$ (real part) & \multicolumn{4}{c}{$\boldsymbol{v}$ (imaginary part) } \\
eta $(\boldsymbol{\eta})$ & $L=6$ & $L=12$ & eta $(\boldsymbol{\eta})$ & $L=6$ & $L=12$ & eta $(\boldsymbol{\eta})$ \\
\hline 0 & 1 & 1 & 0 & 1 & 1 & 0 \\
2 & 0.0268 & 0.0268 & 2 & 0.0268 & 0.0268 & 2 \\
4 & 0.0008932 & 0.0008939 & 4 & 0.0008932 & 0.0008939 & 4 \\
6 & 0 & $2.74 \times 10^{-5}$ & 6 & 0 & $2.74 \times 10^{-5}$ & 6 \\
8 & & $7.88 \times 10^{-7}$ & 8 & & $7.88 \times 10^{-7}$ & 8 \\
10 & & $2.157 \times 10^{-8}$ & 10 & & $2.157 \times 10^{-8}$ & 10 \\
12 & & 12 & & 0 & 12 \\
14 & & & 14 & & & 14 \\
16 & & 16 & & & 16 \\
18 & & 18 & & & 18 \\
\hline
\end{tabular}

\section{Table 2}

The temperature distribution at different lengths by FDM

\begin{tabular}{llll}
\hline & $\theta$ & & \\
eta $(\eta)$ & $L=6$ & eta $(\eta)$ & $L=6$ \\
\hline 0 & 1 & 0 & 1 \\
2 & 0.01208 & 2 & 0.01208 \\
4 & 0.000146 & 4 & 0.000146 \\
6 & 0 & 6 & 0 \\
8 & & 8 & \\
10 & & 10 & \\
12 & & 12 & \\
\hline
\end{tabular}



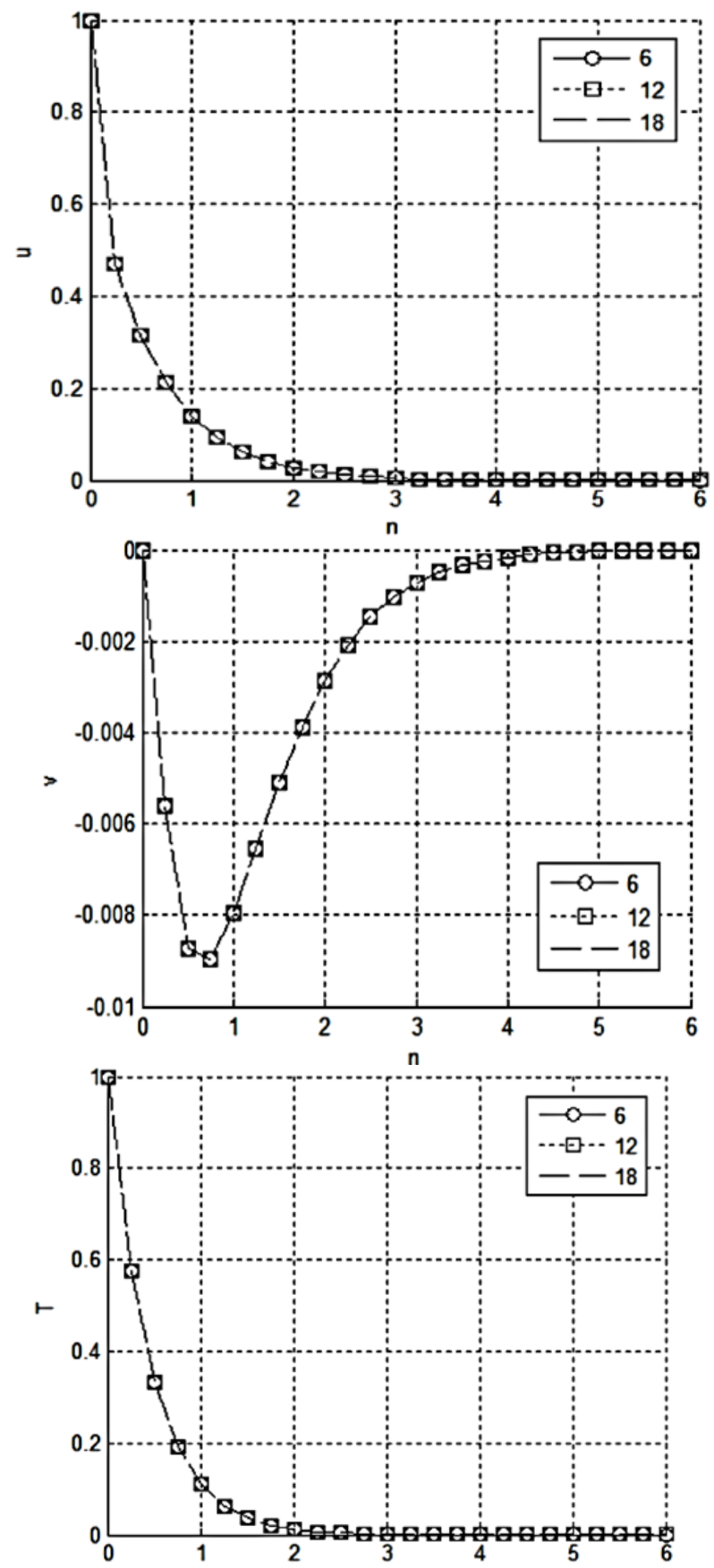

Fig. 1. FDM at $L=L_{1}, L_{2}, L_{3}=6,12,18$ with $a, b, c, \zeta, \Omega, M, G r, \operatorname{Pr}, t=1$ fixed

\subsection{Asymptotic Interpolation Method}

The nonlinear least square follow a special function of $y\left(L_{i}\right)=a_{1}+a_{2} e^{-a_{3}{ }^{2} L_{i}}[25,26]$. MATLAB is used to achieve the best fit of data. Table 3 until Table 5 show the results up to the $L=6$. Using minimisation techniques, coefficient $a=\left(\begin{array}{l}a_{1} \\ a_{2} \\ a_{3}\end{array}\right)$ are found from three nonlinear equations as follow 


$$
\begin{aligned}
& \sum_{i=1}^{3}-2\left(y_{i}-a_{1}-a_{2} e^{-a_{3}^{2} x_{i}}\right)=0 \\
& \sum_{i=1}^{3}-2 e^{-a_{3}^{2} x_{i}}\left(y_{i}-a_{1}-a_{2} e^{-a_{3}^{2} x_{i}}\right)=0 \\
& \sum_{i=1}^{3} 2\left(y_{i}-a_{1}-a_{2} e^{-a_{3}^{2} x_{i}}\right) \cdot 2 a_{3} x_{i}\left(a_{2} e^{-a_{3}^{2} x_{i}}\right)=0
\end{aligned}
$$

The data obtained by the hybrid method shown in Table 3 , Table 4 and Table 5 operating with three different lengths $L=L_{1}, L_{2}, L_{3}=6,12,18$ to define the infinite length. $y_{1}, y_{2}$ and $y_{3}$ are the results at different lengths and $\varepsilon_{i}$ is prediction errors, $\varepsilon_{i}=y_{i}-y(L)=y_{i}-\left(a_{1}+a_{2} e^{-a_{3}^{2} x_{i}}\right)$. The results obtained at each node are similar with $a_{1}$.

Table 3

The real part of velocity profile after asymptotic interpolation method

\begin{tabular}{llllllll}
\hline$\eta$ & $L$ & $y_{1}$ & $y_{2}$ & $y_{3}$ & $a=\left(\begin{array}{l}a_{1} \\
a_{2} \\
a_{3}\end{array}\right)$ & $\varepsilon_{i}$ & $u$ \\
& & & & & & \\
\hline 0 & $L_{1}$ & 1 & 1 & 1 & 1.0000 & & \\
& $L_{2}$ & & 1 & 1 & 0.8504 & $9.728 \times 10^{-8}$ & 1 \\
& $L_{3}$ & & & 1 & 1.1483 & & \\
2 & $L_{1}$ & 0.0268 & 0.0268 & 0.0268 & 0.02680 .85891 .1491 & $9.7297 \times 10^{-8}$ & 0.0268 \\
& $L_{2}$ & 0.0407 & 0.0268 & 0.0268 & & & \\
& $L_{3}$ & 0.0407 & 0.0407 & 0.0268 & & & \\
4 & $L_{1}$ & 0.0009 & 0.0009 & 0.0009 & 0.00090 .85901 .1492 & $9.7529 \times 10^{-8}$ & 0.0009 \\
& $L_{2}$ & 0.0014 & 0.0009 & 0.0009 & & & \\
& $L_{3}$ & 0.0014 & 0.0014 & 0.0009 & & & \\
6 & $L_{1}$ & 0.0000 & 0.0000 & 0.0000 & 0.00000 .85611 .1521 & $1.0589 \times 10^{-7}$ & 0.0000 \\
& $L_{2}$ & 0.0000 & 0.0000 & 0.0000 & & & \\
& $L_{3}$ & 0.0000 & 0.0000 & 0.0000 & & & \\
\hline
\end{tabular}

Table 4

The imaginary part of velocity profile after asymptotic interpolation method

\begin{tabular}{llllllll}
\hline$\eta$ & $L$ & $y_{1}$ & $y_{2}$ & $y_{3}$ & $a=\left(\begin{array}{l}a_{1} \\
a_{2} \\
a_{3}\end{array}\right)$ & $\varepsilon_{i}$ & $u$ \\
& & & & & & \\
\hline 0 & $L_{1}$ & 0 & 0 & 0 & 0.0000 & $9.729 \times 10^{-8}$ & 0.0000 \\
& $L_{2}$ & & 0 & 0 & 0.8591 & & \\
& $L_{3}$ & & & 0 & 1.1491 & & \\
2 & $L_{1}$ & -0.0029 & -0.0029 & -0.0029 & -0.0029 & $9.7288 \times 10^{-8}$ & -0.0029 \\
& $L_{2}$ & -0.0039 & -0.0029 & -0.0029 & 0.8592 & & \\
& $L_{3}$ & -0.0039 & -0.0039 & -0.0029 & 1.1491 & & \\
4 & $L_{1}$ & -0.0002 & -0.0002 & -0.0002 & -0.0002 & $9.7225 \times 10^{-8}$ & -0.0002 \\
& $L_{2}$ & -0.0002 & -0.0002 & -0.0002 & 0.8592 & & \\
& $L_{3}$ & -0.0002 & -0.0002 & -0.0002 & 1.1490 & & \\
6 & $L_{1}$ & 0.0000 & 0.0000 & 0.0000 & -0.0000 & $9.5355 \times 10^{-8}$ & -0.0000 \\
& $L_{2}$ & 0.0000 & 0.0000 & 0.0000 & 0.8599 & & \\
& $L_{3}$ & 0.0000 & 0.0000 & 0.0000 & 1.1484 & & \\
\hline
\end{tabular}




\section{Table 5}

The temperature distribution after asymptotic interpolation method

\begin{tabular}{|c|c|c|c|c|c|c|c|}
\hline$\eta$ & $L$ & $y_{1}$ & $y_{2}$ & $y_{3}$ & $a=\left(\begin{array}{l}a_{1} \\
a_{2} \\
a_{3}\end{array}\right)$ & $\varepsilon_{i}$ & $\theta$ \\
\hline \multirow[t]{3}{*}{0} & $L_{1}$ & 1 & 1 & 1 & 1.0000 & $9.728 \times 10^{-8}$ & 1 \\
\hline & $L_{2}$ & & 1 & 1 & 0.8504 & & \\
\hline & $L_{3}$ & & & 1 & 1.1483 & & \\
\hline \multirow[t]{3}{*}{2} & $L_{1}$ & 0.0121 & 0.0121 & 0.0121 & \multirow[t]{3}{*}{0.01210 .85901 .1491} & $9.729 \times 10^{-8}$ & 0.0121 \\
\hline & $L_{2}$ & 0.0120 & 0.0121 & 0.0121 & & & \\
\hline & $L_{3}$ & 0.0120 & 0.0120 & 0.0121 & & & \\
\hline \multirow[t]{3}{*}{4} & $L_{1}$ & 0.0001 & 0.0001 & 0.0001 & \multirow[t]{3}{*}{0.00010 .85911 .1491} & $9.7297 \times 10^{-8}$ & 0.0001 \\
\hline & $L_{2}$ & 0.0003 & 0.0001 & 0.0001 & & & \\
\hline & $L_{3}$ & 0.0003 & 0.0003 & 0.0001 & & & \\
\hline \multirow[t]{3}{*}{6} & $L_{1}$ & 0.0000 & 0.0000 & 0.0000 & \multirow[t]{3}{*}{0.00000 .85891 .1493} & $9.7826 \times 10^{-8}$ & 0.0000 \\
\hline & $L_{2}$ & 0.0000 & 0.0000 & 0.0000 & & & \\
\hline & $L_{3}$ & 0.0000 & 0.0000 & 0.0000 & & & \\
\hline
\end{tabular}

Table 6 shows the comparison results from FDM and hybrid method of finite difference asymptotic interpolation with all parameters set to $a, b, c, \zeta, \Omega, M, G r, \operatorname{Pr}, t=1$. The output from FDM clearly show that the terminal node received zero value but not in hybrid method. The results of the hybrid method show that as length increases, the velocity profile and temperature increase and approaching zero which is satisfied the boundary condition of $f(\eta, \tau) \rightarrow 0$ as $\eta \rightarrow \infty$ This findings present that the hybrid method is better compared to FDM.

\section{Table 6}

Comparison of velocity profile by FDM and hybrid method

\begin{tabular}{lllllll}
\hline eta $(\eta)$ & $u$ & & $v$ & & $\theta$ & \\
& FDM & Hybrid Method & FDM & Hybrid Method & FDM & Hybrid Method \\
\hline 0 & 1 & 1 & 0 & 0 & 1 & 1 \\
1 & 0.1406 & 0.1406 & -0.007967 & -0.007966 & 0.1099 & 0.1099 \\
2 & 0.0268 & 0.0268 & -0.002858 & -0.002858 & 0.01208 & 0.01208 \\
3 & 0.004955 & 0.004956 & $-7.319 \times 10^{-4}$ & $-7.316 \times 10^{-4}$ & 0.001328 & 0.001329 \\
4 & 0.0008932 & 0.0008943 & $-1.624 \times 10^{-4}$ & $-1.622 \times 10^{-4}$ & 0.000146 & 0.0001464 \\
5 & 0.0001532 & 0.0001583 & $-3.206 \times 10^{-5}$ & $-3.288 \times 10^{-5}$ & $1.586 \times 10^{-5}$ & $1.643 \times 10^{-5}$ \\
6 & 0 & $2.779 \times 10^{-5}$ & 0 & $-6.065 \times 10^{-6}$ & 0 & $-2.147 \times 10^{-6}$ \\
\hline
\end{tabular}

\subsection{Validation With HAM}

The validation of the hybrid method is carried out by comparing the result with Nazari [29] without heat transfer. Figure 2(a) depicts the real and imaginary parts of the velocity profile obtained from HAM. Meanwhile Figure 2(b) depicts the velocity profile from the present study, which has been obtained using a hybrid method. This graph shows that the velocity profiles for both methods increase as time increases. 

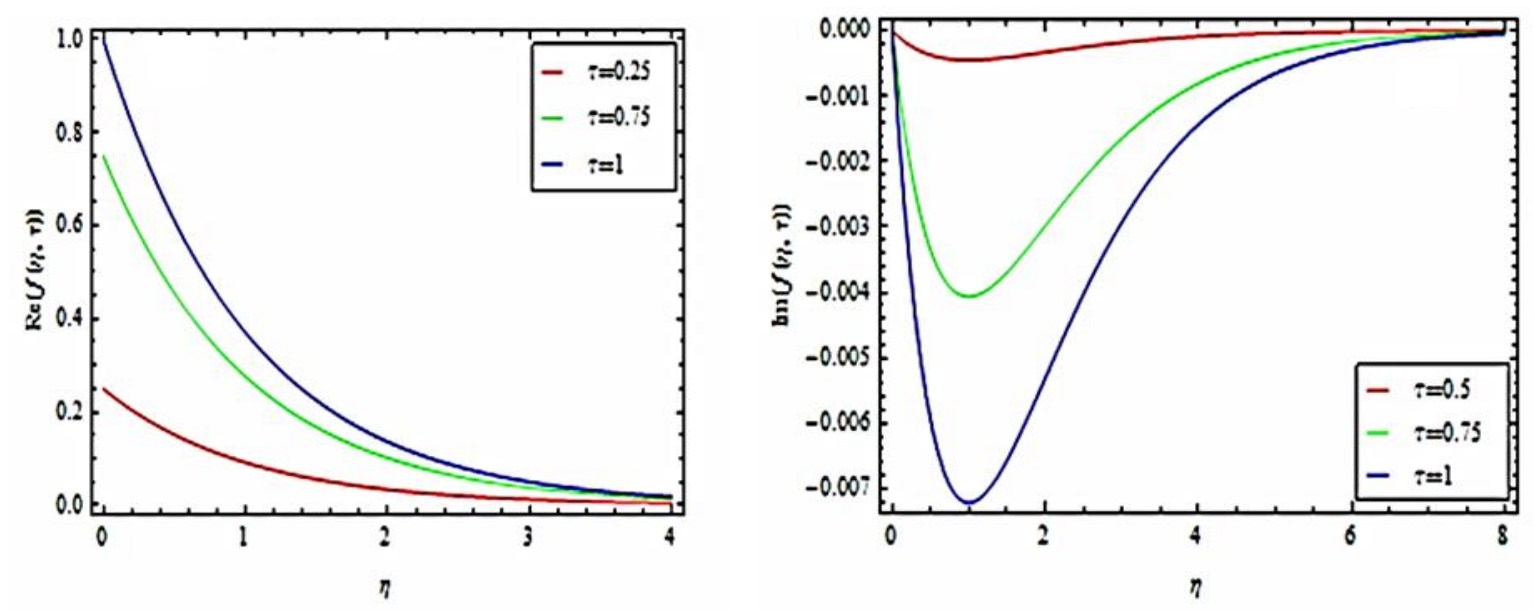

(a)
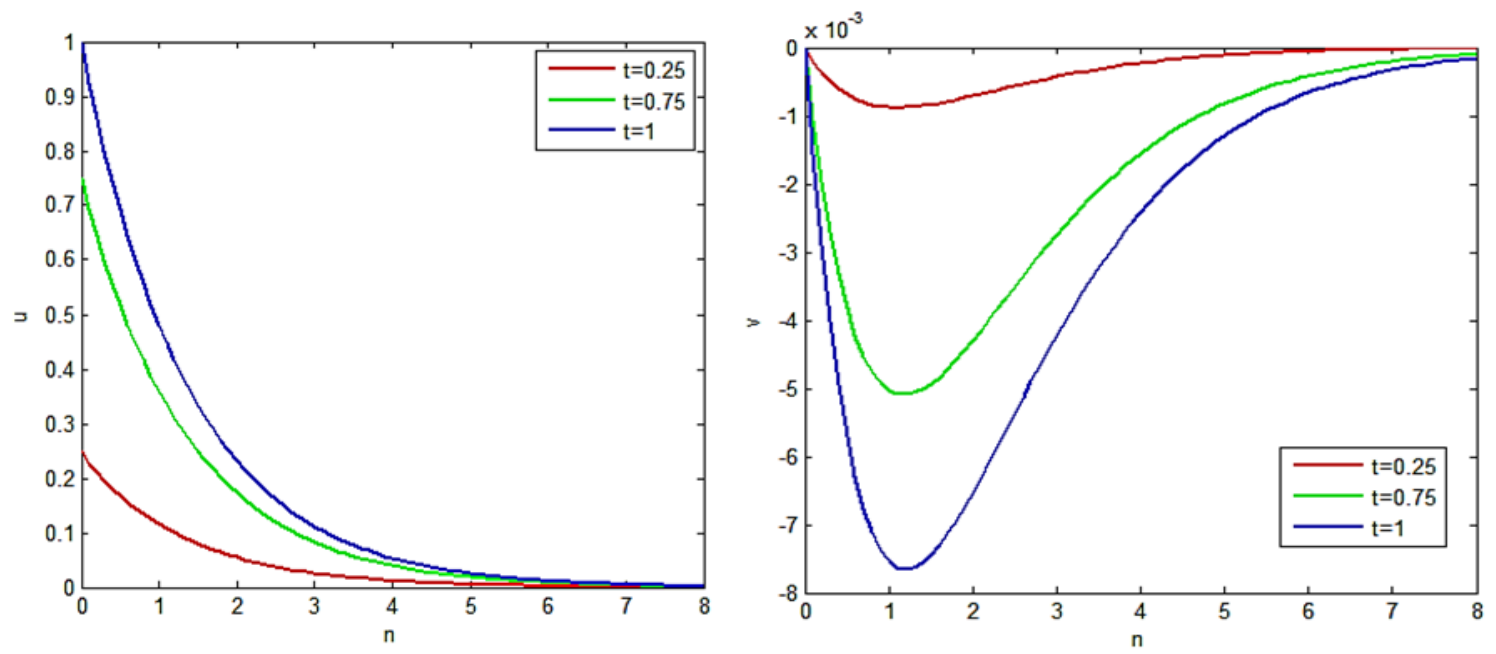

(b)

Fig. 2. Comparison of velocity profile for different value of time, $\tau$ with others parameter fixed (a) HAM (Nazari [29]) (b)Hybrid method (present work)

The velocity profile from HAM is also shown in Figure 3(a), and the current results from the hybrid method are shown in Figure 3(b). The figures seem to have the same curvature pattern. With an increasing in the third-grade parameter, the velocity profile increases.
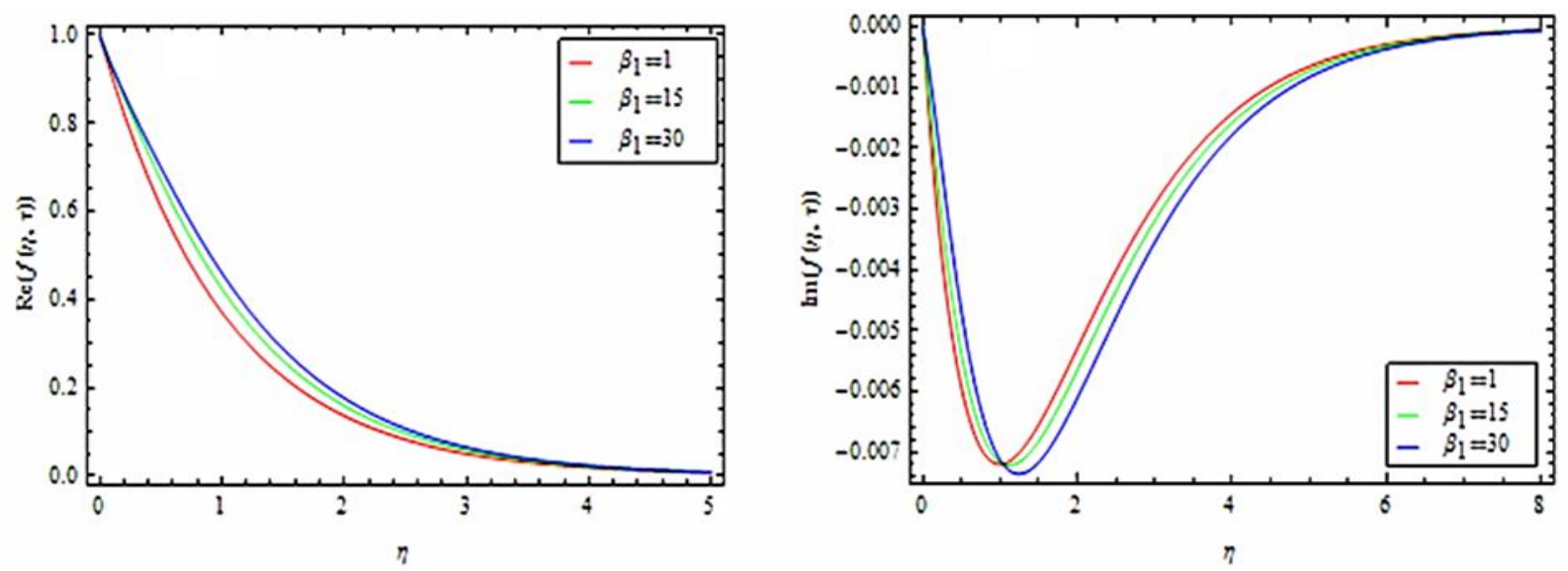

(a) 

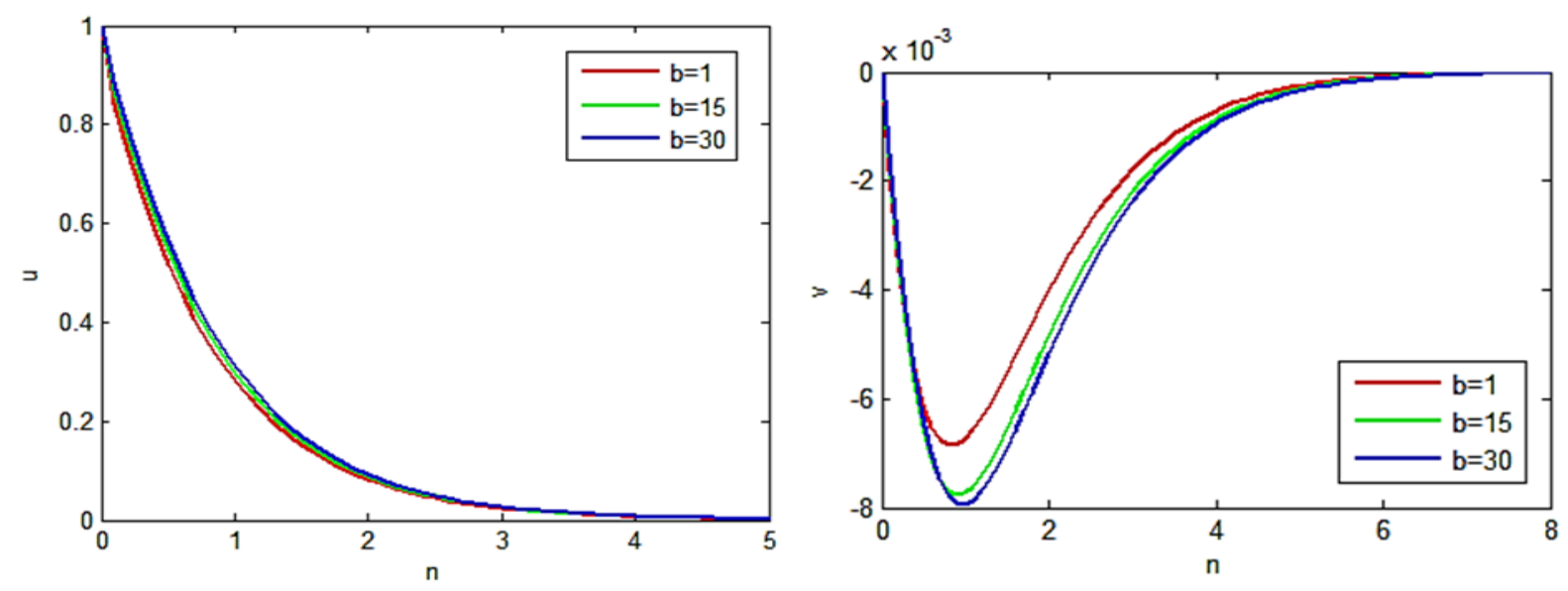

(b)

Fig. 3. Comparison of velocity profile for different value of third grade fluid, $b$ with others parameter fixed (a) HAM (Nazari [29]) (b)Hybrid method (present work)

\section{Results and Findings}

MATLAB Software with Intel(R) Core (TM) i7 processor was used to illustrate the findings. Different values of parameter were tested. Figure 4 depicts the velocity distribution at the real and imaginary parts for various porosity parameter values. The results show that as $c$ increases from 1 to 15 , the velocity decreases. This result is similar with Zeeshan [30], who discovered that increasing porosity reduces fluid velocity. This is related to permeability, where the fluid can quickly move through the medium with large pores. According to Billen [31], the loose medium has greater permeability than the consolidated medium.
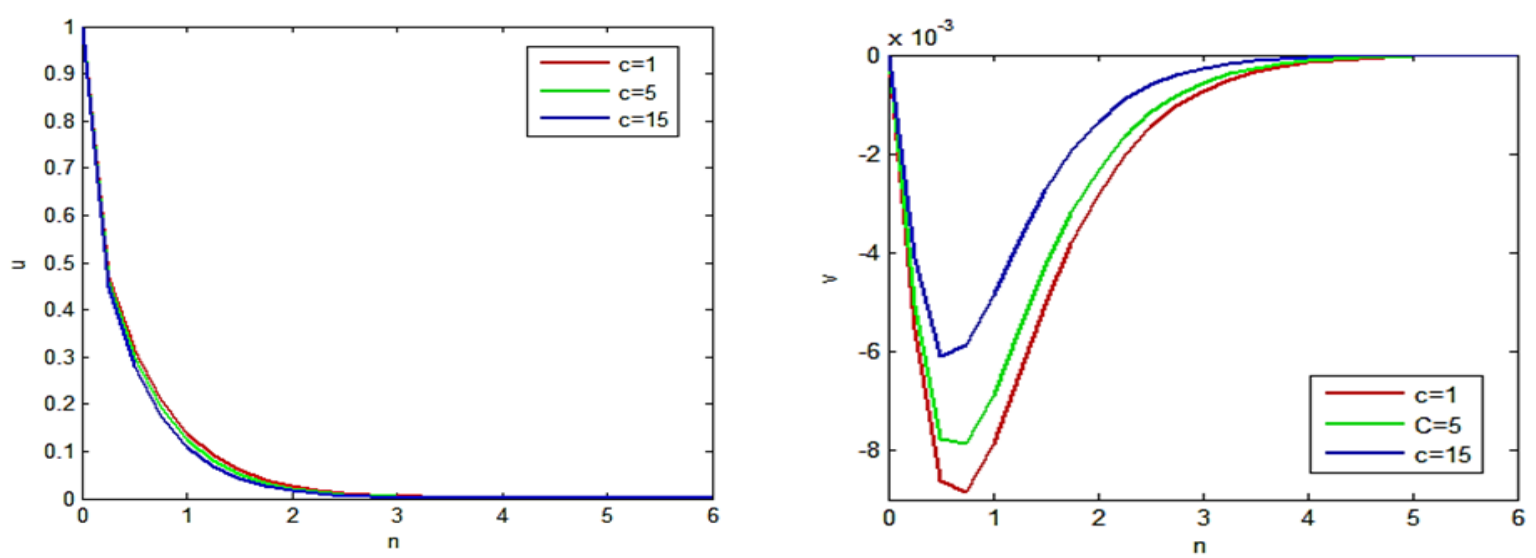

Fig. 4. Velocity profile for different value of porous parameter, $c$ with others parameter fixed at $a, b, \zeta, \Omega, M, G r, \operatorname{Pr}, t=1$

In this fluid issue, the value of the Grashof number is chosen to be $G r>0$, which corresponds to fluid medium cooling, as highlighted by Nayak [8]. Figure 5 depicts the results for various Grashof number values. A large number of $G r$ decreases kinematic viscosity and thus increases the velocity profile's boundary layer. Anita [12] investigated the impact of different values of $G r$ and reported to a similar conclusion. It should be noted that for $\operatorname{Pr}=1$, both heat and momentum dissolve over the fluid in about the same ratio. 

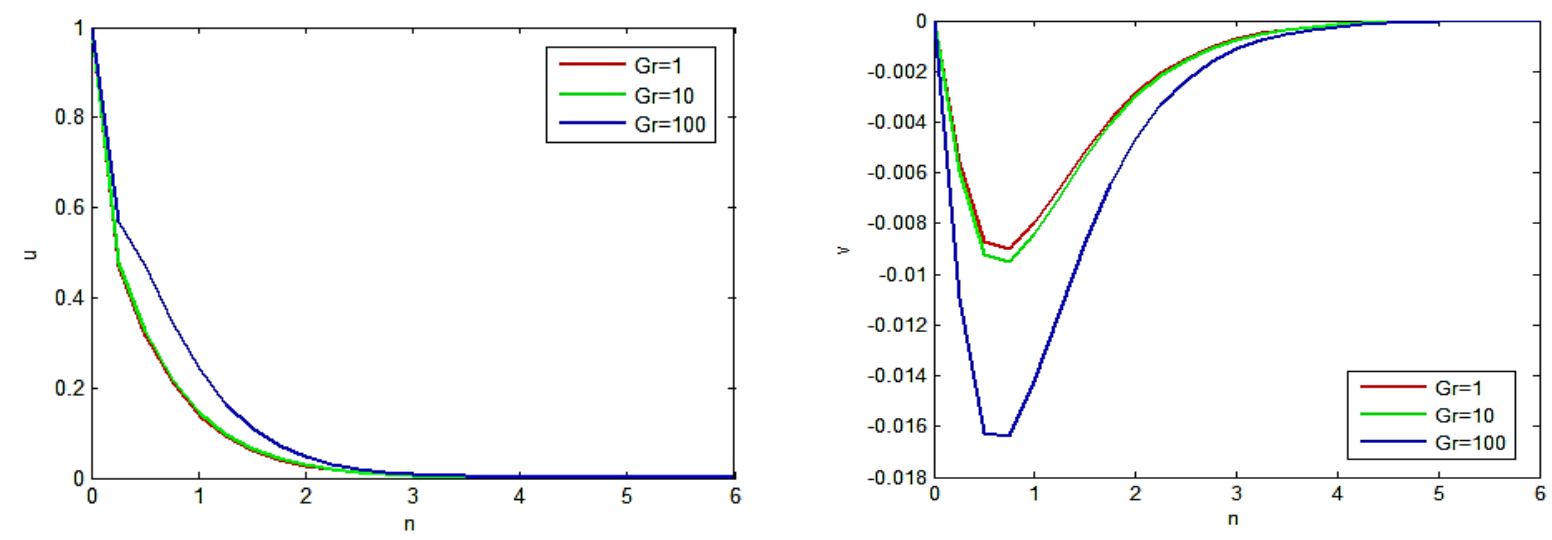

Fig. 5. Velocity profile for different value of Grashoff number, $G r$ with others parameter fixed at $a, b, c, \zeta, \Omega, M, \operatorname{Pr}, t=1$

Figure 6 depicts the relationship between the Grashof number, $G r$ and the Prandtl numbers, $P r$. Four $\mathrm{Gr}$ and $\mathrm{Pr}$ couple sets are investigated. $\mathrm{Pr}<1$ shows that thermal diffusivity is the dominant factor. As a result, for every value of $G r$ the temperature distribution increased. In this case, heat conduction happens. The thickness of the thermal boundary layer is greater than that of the velocity boundary layer. A liquid metal, such as mercury, is a typical example of this fluid. Meanwhile, $\operatorname{Pr}>1$ indicates that momentum diffusivity is dominant and essential to the convection phase. As a result, the thermal boundary layer becomes thinner. However, it is also shown that for $G r>\operatorname{Pr}$, the boundary layer is thicker than for $G r<P r$. Furthermore, it is observed that as $P r$ increases, the temperature decreases [32,33].

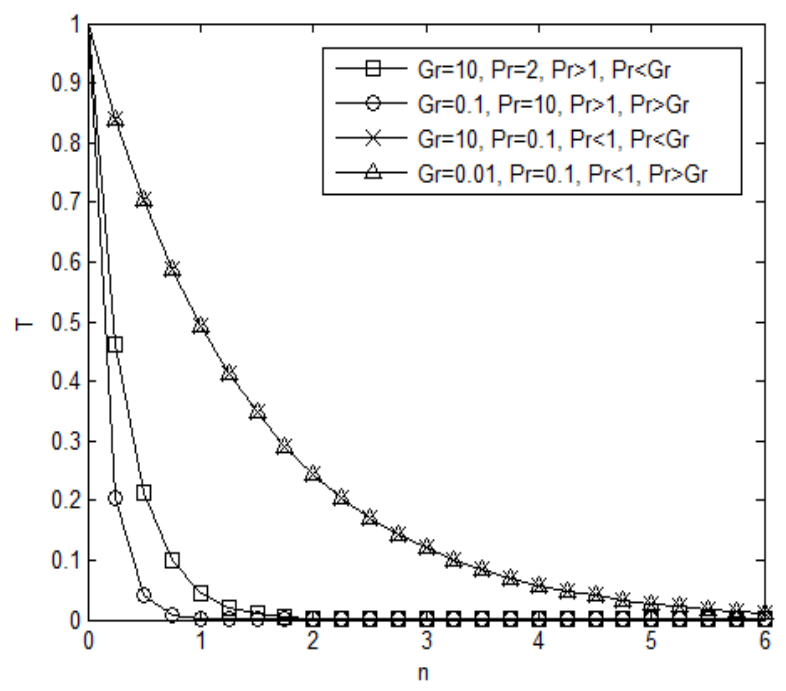

Fig. 6. Effect of $\mathrm{Gr}$ and $\mathrm{Pr}$ towards the temperature distribution

Figure 7 depicts the effect of a magnetic field on the velocity of a fluid. Physically, as the magnetic parameter increases from 5 to 15 the Lorentz force emerges and slows the fluid motion [34]. The fluid's velocity decreases because of this condition. The effect of different values of second- and thirdgrade parameters on the velocity profile of fluid flow is investigated. Figure 8 shows that increasing the non-Newtonian parameter $a$ leads to increase in velocity. This result is consistent with the results of previous research by Siddiqui [35]. Figure 9 also shows that increasing the parameter $b$ cause 
velocity to increase. Figure 10 also presents the relationship between time and velocity profile are directly proportional where velocity increase when time increase.
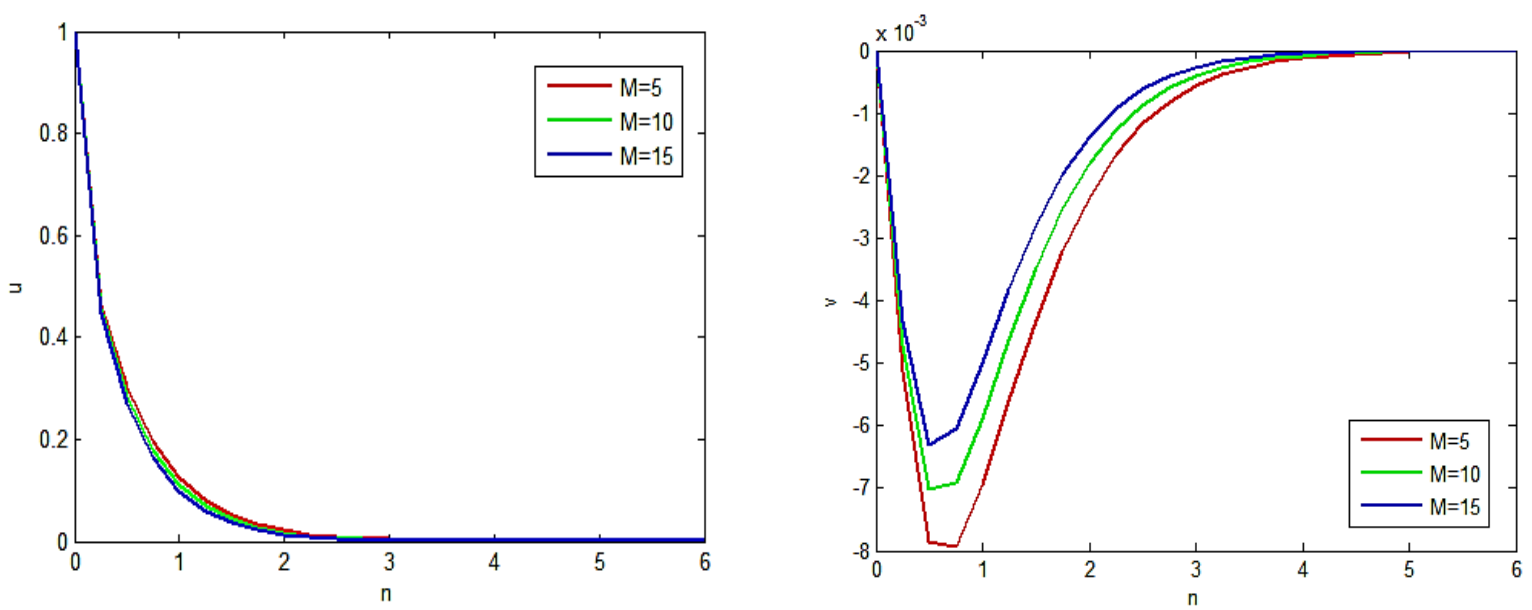

Fig. 7. Velocity profile for different value of magnetic parameter, $M$ with others parameter fixed at $a, b, c, \zeta, \Omega, G r, \operatorname{Pr}, t=1$
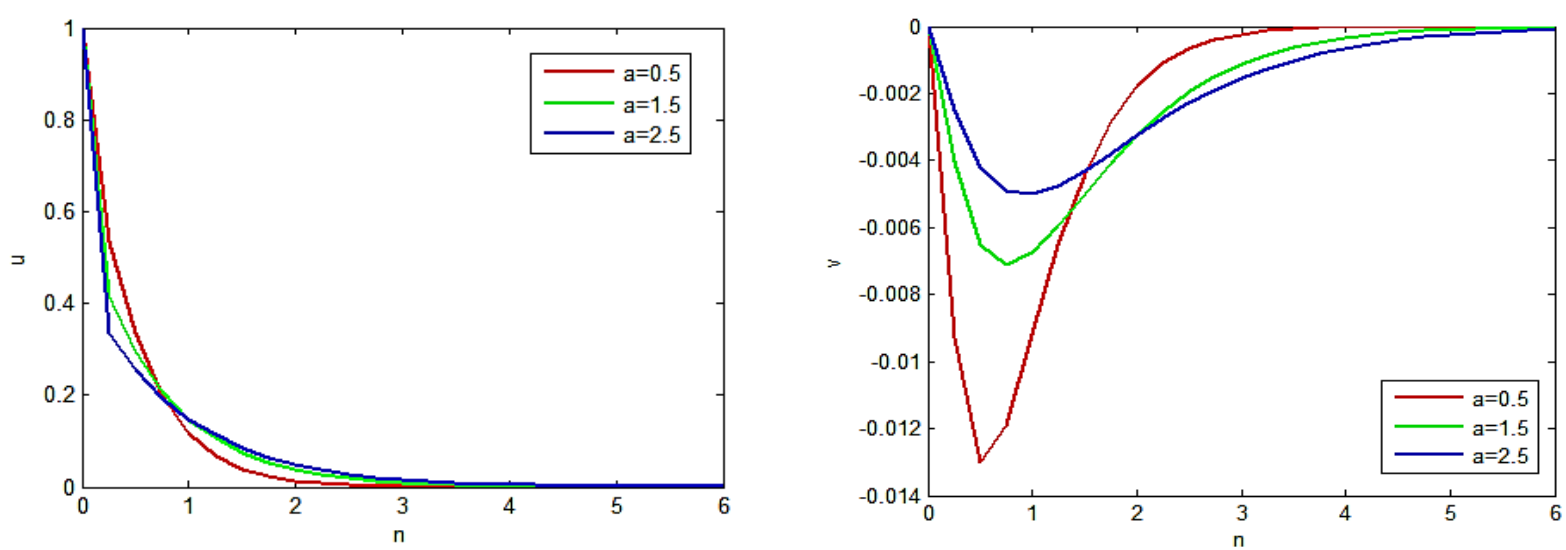

Fig. 8. Velocity profile for different value of second-grade parameter, $a$ with others parameter fixed at $b, c, \zeta, M, \Omega, G r, \operatorname{Pr}, t=1$
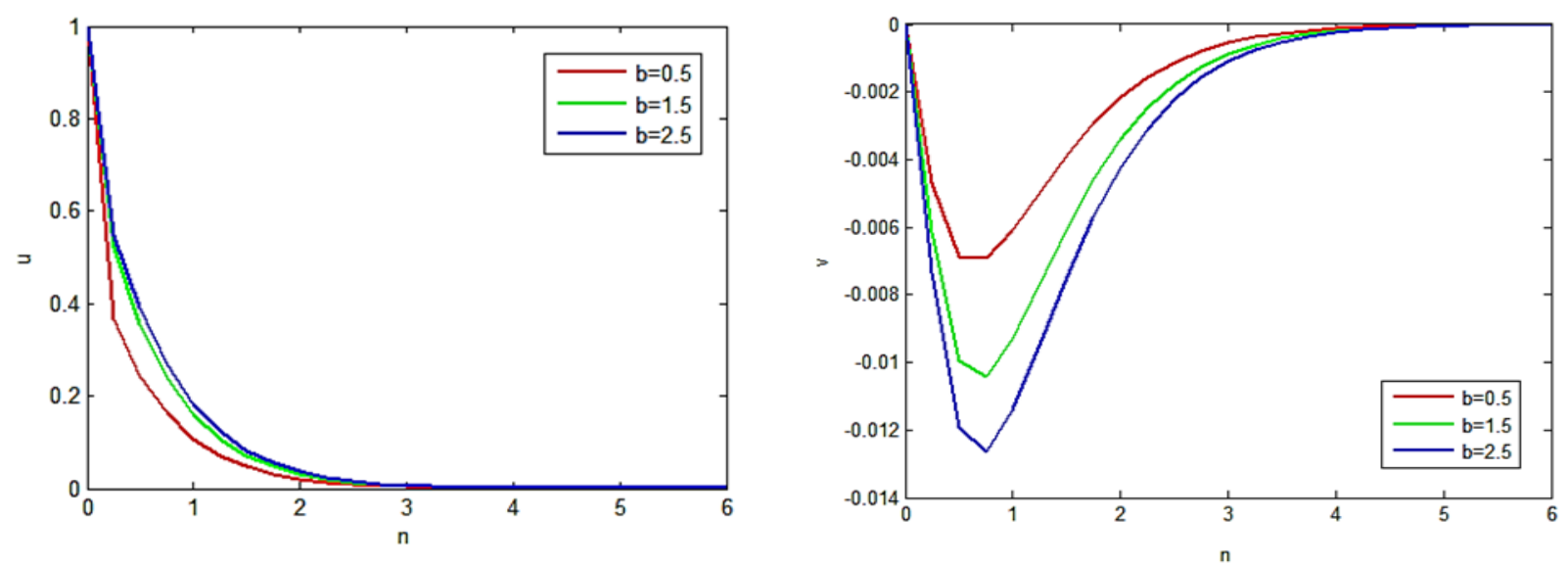

Fig. 9. Velocity profile for different value of third-grade parameter, $b$ with others parameter fixed at $a, c, \zeta, M, \Omega, G r, \operatorname{Pr}, t=1$ 

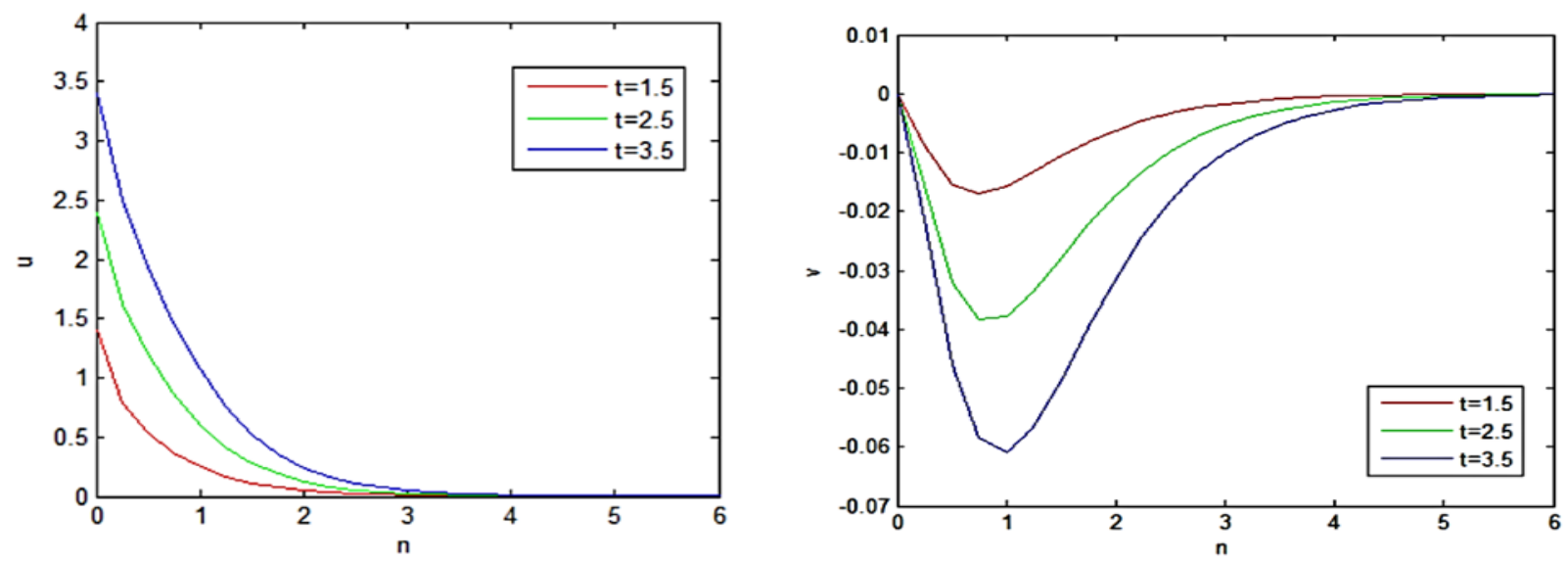

Fig. 10. Velocity profile for different value of third-grade parameter, $t$ with others parameter fixed at $a, b, c, \zeta, M, \Omega, G r, \operatorname{Pr}=1$

\section{Discussions}

The third-grade fluid flows in a rotating frame through a magnetic field and a porous medium have been analysed. In order to address the fluid flow problem, a hybrid method of finite difference - asymptotic interpolation is adopted. To fulfil the infinite size of the domain, this hybrid method used three different lengths in FDM as shown in Figure 1 , Table 1 and Table 2, which had been later combined with an asymptotic function $y=a_{1}+a_{2} e^{-a_{3}{ }^{2} L}[25,26]$. The best fit is achieved by using a non-linear least square curve fit. The hybrid process solution is $a_{1}[25,26]$. Table 3 until Table 5 indicate the hybrid solution and how the outcomes met the condition in Eq. (16). There is no exact solution to this problem that has been published. The issue has been compared with semi-analytical HAM in the absence of heat transfer, as shown in Figure 2 and Figure 3 .

Heat transfer is assigned to the fluid flow problem in the following section. The lengths of the problem $L=L_{1}, L_{2}, L_{3}=6,12,18$ are chosen to present the limiting case $\eta \rightarrow \infty$. Table 6 shows that the outputs of the hybrid method are larger than the outputs of the finite difference method. Various values of the porosity parameter, Grashof number, PrandtI number, MHD parameter, time, secondand third-grade parameters are tested to see how they influence the velocity and temperature distribution. The outcomes are depicted in Figure 4 until Figure 10. The fluid velocity decrease as the porosity parameter increased. Increasing the Grashof number, on the other hand, will boost the buoyancy force of the flow and leads to increase the velocity profile [33]. Figure 4 describes the relationship between Prandtl and Grashof numbers. Temperature goes up for a low PrandtI number $(\operatorname{Pr}<1)$. The fluid flow in a magnetic field. As a result, the higher the MHD, the slower the velocity. The results are consistent with previous studies.

\section{Conclusions}

The problem of unsteady constant acceleration MHD third-grade fluid flow in a rotating frame through porous medium with a presence of heat transfer is conducted. A new hybrid method of finite difference method and asymptotic interpolation method is adopted to deal with the semi-infinite size of the fluid flow problem. The procedure of method is shown in separate sections with all the parameters fixed; $a, b, c, \zeta, \Omega, M, G r, P r, t=1$. The advantage of the hybrid method over FDM is presented. It reveals that the results from the hybrid method are greater and fulfil the boundary condition compared to the FDM that presents zero result at infinite length. The result is validated 
with HAM. Various parameters with different value are tested and the findings show that there is relation between parameters with velocity and temperature distribution.

\section{Acknowledgement}

Researchers from Universiti Teknologi MARA, Johor Branch, Universiti Teknologi Malaysia, Skudai, and the University of Kordofan, Sudan collaborated on this research.

\section{References}

[1] Salah, Faisal, Zainal Abdul Aziz, and Dennis Ling Chuan Ching. "On accelerated MHD flows of second grade fluid in a porous medium and rotating frame." IAENG International Journal of Applied Mathematics 43, no. 3 (2013): 106113. https://doi.org/10.1155/2013/485805

[2] Salah, Faisal, Zainal Abdul Aziz, and Dennis Ling Chuan Ching. "New exact solutions for MHD transient rotating flow of a second-grade fluid in a porous medium." Journal of Applied Mathematics 2011 (2011). https://doi.org/10.1155/2011/823034

[3] Abelman, S., E. Momoniat, and T. Hayat. "Steady MHD flow of a third grade fluid in a rotating frame and porous space." Nonlinear Analysis: $\quad$ Real World Applications 10, no. $6 \quad$ (2009): 3322-3328. https://doi.org/10.1016/i.nonrwa.2008.10.067

[4] Sadd, M. H. "Chapter 5-Formulation and Solution Strategies." Elasticity, Theory, Applications, and Numerics; Sadd, MH, Ed: 83-102. https://doi.org/10.1016/B978-012605811-6/50006-3

[5] Blazek, Jiri. "Principles of solution of the governing equations." Computational Fluid Dynamics: Principles and Applications, 3rd ed.; Blazek, J., Ed (2015): 121-166. https://doi.org/10.1016/B978-0-08-099995-1.00005-1

[6] Tomé, Murilo F., Brian Duffy, and Sean McKee. "A numerical technique for solving unsteady non-Newtonian free surface flows." Journal of Non-Newtonian Fluid Mechanics 62, no. 1 (1996): 9-34. https://doi.org/10.1016/0377$\underline{0257(95) 01391-1}$

[7] Tomé, Murilo F., L. Grossi, Antonio Castelo, José A. Cuminato, Norberto Mangiavacchi, Valdemir G. Ferreira, F. S. De Sousa, and Sean McKee. "A numerical method for solving three-dimensional generalized Newtonian free surface flows." Journal of Non-Newtonian Fluid Mechanics 123, no. 2-3 (2004): 85-103. https://doi.org/10.1016/i.jnnfm.2004.06.012

[8] Nayak, Itishree, Ajit Kumar Nayak, and Sudarsan Padhy. "Numerical solution for the flow and heat transfer of a third grade fluid past a porous vertical plate." Adv. Stud. Theor. Phys 6, no. 13 (2012): 615-625.

[9] Rana, M. A., Akhlaq Ahmed, and Rashid Qamar. "Magnetohydrodynamic Rotating Flow of a Fourth Grade Fluid Between Two Parallel Infinite Plates." Topics in Magnetohydrodynamics (2012): 189. https://doi.org/10.5772/37410

[10] Parida, Mamata, and Sudarsan Padhy. "Numerical study of MHD flow of a third grade fluid in a non-Darcian porous channel." International Journal of Pure and Applied Mathematics 118, no. 3 (2018): 651-665.

[11] Garg, B. P., Singh, K. D. and Neeraj, "Chemically Reacting, Radiating and Rotating MHD Convective Flow of VicoElastic Fluid through Porous Medium in Vertical Channel. " International Journal of Latest Trends in Engineering and Technology 5, no. 2 (2015).

[12] Anitha, K. "Numerical solution of an unsteady MHD flow of a rotating fluid past an infinite vertical porous plate in the presence of radiation and chemical reaction." Electronic Journal of Mathematical Analysis and Applications 3, no. 2 (2015): 202-214.

[13] Nayak, Itishree. "Numerical study of MHD flow and heat transfer of an unsteady third grade fluid with viscous dissipation." IAENG International Journal of Applied Mathematics 49, no. 2 (2019): 1-8.

[14] Katagi, Nagaraj N., and Ashwini Bhat. "Finite Difference Solution for MHD Flow Between Two Parallel Permeable Plates with Velocity Slip." Journal of Advanced Research in Fluid Mechanics and Thermal Sciences 76, no. 3 (2020): 38-48. https://doi.org/10.37934/arfmts.76.3.3848

[15] Bhat, Ashwini, and Nagaraj N. Katagi. "Magnetohydrodynamic flow of micropolar fluid and heat transfer between a porous and a non-porous disk." Journal of Advanced Research in Fluid Mechanics and Thermal Sciences 75, no. 2 (2020): 59-78. https://doi.org/10.37934/arfmts.75.2.5978

[16] Makhija, S. U. M. A. N. "Some Stability Problems of Non-Newtonian Fluids." Synopsis of the thesis for the Degree of Philosophy, Department of Mathematics, Jaypee Institute of Information Technology, A-10, Sector-62, Noida, India (2012).

[17] Hayat, T., C. Fetecau, and M. Sajid. "Analytic solution for MHD transient rotating flow of a second grade fluid in a porous space." Nonlinear Analysis: Real World Applications 9, no. $4 \quad$ (2008): 1619-1627. https://doi.org/10.1016/i.nonrwa.2007.04.006 
[18] Abdul Aziz, Zainal, Mojtaba Nazari, Faisal Salah, and Dennis Ling Chuan Ching. "Constant accelerated flow for a third-grade fluid in a porous medium and a rotating frame with the homotopy analysis method." Mathematical Problems in Engineering 2012 (2012). https://doi.org/10.1155/2012/601917

[19] Hayat, Tasawar, Rahmat Ellahi, and Fazal Mehmood Mahomed. "The analytical solutions for magnetohydrodynamic flow of a third order fluid in a porous medium." Zeitschrift für Naturforschung A 64, no. 9-10 (2009): 531-539. https://doi.org/10.1515/zna-2009-9-1001

[20] Aiyesimi, Y. M., G. T. Okedayo, and O. W. Lawal. "Unsteady magnetohydrodynamic (MHD) thin film flow of a third grade fluid with heat transfer and no slip boundary condition down an inclined plane." International journal of physical sciences 8, no. 19 (2013): 946-955. https://doi.org/10.5897/IJPS2013.3891

[21] El-dabe, Nabil Tawfik, Mohamed Yahya Abou-zeid, and Ola S. Ahmed. "Motion of a thin film of a fourth grade nanofluid with heat transfer down a vertical cylinder: Homotopy perturbation method application." Journal of Advanced Research in Fluid Mechanics and Thermal Sciences 66, no. 2 (2020): 101-113.

[22] Andrianov, Igor V., and Jan Awrejcewicz. "New trends in asymptotic approaches: summation and interpolation methods." Appl. Mech. Rev. 54, no. 1 (2001): 69-92. https://doi.org/10.1115/1.3097289

[23] Vyaz'min, A. V., I. A. Denisov, and A. D. Polyanin. "Method of asymptotic interpolation in problems of chemical hydrodynamics and mass transfer." Theoretical Foundations of Chemical Engineering 35, no. 1 (2001): 1-8. https://doi.org/10.1023/A:1005252031967

[24] Yukalov, V. I., E. P. Yukalova, and S. Gluzman. "Extrapolation and interpolation of asymptotic series by self-similar approximants." Journal of mathematical chemistry 47, no. 3 (2010): 959-983. https://doi.org/10.1007/s10910-0099618-1

[25] Mahadi, S., F. Salah, N. Arbin, and S. H. Yeak. "Hybrid numerical solution for unsteady state of constant accelerated MHD in a third-grade fluid with a rotation." In Journal of Physics: Conference Series, vol. 1489, no. 1, p. 012007. IOP Publishing, 2020. https://doi.org/10.1088/1742-6596/1489/1/012007

[26] Mahadi, Shafaruniza, Zainal Abdul Aziz, Yeak Su Hoe, Faisal Salah, and Farah Suraya Md Nasrudin. "Numerical solution of hybrid method for third grade flow due to variable accelerated plate in a rotating frame." International Journal of Engineering and Technology 7, no. 2.15 (2018): 98-101. https://doi.org/10.14419/ijet.v7i2.15.11361

[27] Hayat, Tasawar, and A. H. Kara. "Couette flow of a third-grade fluid with variable magnetic field." Mathematical and Computer Modelling 43, no. 1-2 (2006): 132-137. https://doi.org/10.1016/i.mcm.2004.12.009

[28] Hayat, Tasawar, and Yongqi Wang. "Magnetohydrodynamic flow due to noncoaxial rotations of a porous disk and a fourth-grade fluid at infinity." Mathematical Problems in Engineering 2003, no. 2 (2003): 47-64. https://doi.org/10.1155/S1024123X03308026

[29] Nazari, Mojtaba. "Approximate analytical solutions for viscoelastic differential type flow models using homotopy analysis method." PhD diss., Universiti Teknologi Malaysia, 2014.

[30] Zeeshan, A., R. Ellahi, and M. Hassan. "Magnetohydrodynamic flow of water/ethylene glycol based nanofluids with natural convection through a porous medium." The European Physical Journal Plus 129, no. 12 (2014): 1-10. https://doi.org/10.1140/epjp/i2014-14261-5

[31] Billen, M. "Darcy's Law - Flow in a Porous Medium." University of California, Davis, 2021.

[32] Seth, G. S., Sarkar, S. and Nandkeolyar, R. "Unsteady Hydromagnetic Natural Convection Flow past an Impulsively Moving Vertical Plate with Newtonian Heating in a Rotating System." Journal of Applied Fluid Mechanics 8, no. 3 (2015): 623-633. https://doi.org/10.18869/acadpub.jafm.67.222.22724

[33] Shahrim, Muhammad Nazirul, Ahmad Qushairi Mohamad, Lim Yeou Jiann, Muhamad Najib Zakaria, Sharidan Shafie, Zulkhibri Ismail, and Abdul Rahman Mohd Kasim. "Exact Solution of Fractional Convective Casson Fluid Through an Accelerated Plate." CFD Letters 13, no. 6 (2021): 15-25. https://doi.org/10.37934/cfdl.13.6.1525

[34] Arifuzzaman, S. M., Md Shakhaoath Khan, Abdullah Al-Mamun, Sk Reza-E-Rabbi, Pronab Biswas, and Ifsana Karim. "Hydrodynamic stability and heat and mass transfer flow analysis of MHD radiative fourth-grade fluid through porous plate with chemical reaction." Journal of King Saud University-Science 31, no. 4 (2019): 1388-1398. https://doi.org/10.1016/i.jksus.2018.12.009

[35] Siddiqui, Abdul Majeed, Muhammad Afzal Rana, and Nosheen Zareen Khan. "Effect of a time dependent stenosis on flow of a second grade fluid through porous medium in constricted tube using integral method." Mathematical Sciences 11, no. 4 (2017): 275-285. https://doi.org/10.1007/s40096-017-0229-x 